\title{
Study of Niobium Thin Films under Pressure
}

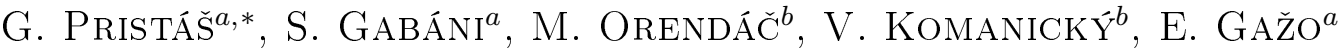 \\ ${ }^{a}$ Institute of Experimental Physics, Slovak Academy of Sciences, Watsonova 47, 04001 Košice, Slovakia \\ ${ }^{b}$ Faculty of Science, P. J. Šafárik University, Park Angelinum 9, 04154 Košice, Slovakia
}

\begin{abstract}
Niobium is widely used in many important superconducting applications. At ambient pressure, bulk Nb has the highest critical temperature, $T_{c} \approx 9.25 \mathrm{~K}$ among the superconducting elements. Thin films of Nb show several differences in behavior in comparison with bulk $\mathrm{Nb}$, e.g. substantial increase in the upper critical field $\left(H_{C 2}\right)$. Critical temperature of superconducting transition is usually lower for thin films than in bulk sample and depends on thickness of the film, size of grains etc. We prepared $100 \mathrm{~nm}$ thick niobium thin films in the high vacuum DC magnetron sputtering system, with $T_{c}=8.95 \mathrm{~K}$ at ambient pressure. In this study, we performed measurements of superconducting transition temperature by electrical resistivity measurements of $\mathrm{Nb}$ thin film under hydrostatic pressure of up to $30 \mathrm{kbar}$. We observed an increase of $T_{c}$ with increasing value of pressure $\left(\mathrm{d} T_{c} / \mathrm{d} p=7.3 \mathrm{mK} / \mathrm{kbar}\right)$. On the other side in the case of bulk sample of $\mathrm{Nb}$ we observed a decrease of $T_{c}$ value $\left(\mathrm{d} T_{c} / \mathrm{d} p=-2.5 \mathrm{mK} / \mathrm{kbar}\right)$ with increasing applied pressure. Difference in superconducting properties between niobium bulk and thin film under pressure is discussed.
\end{abstract}

DOI: 10.12693 /APhysPolA.126.346

PACS: 74.78.-w, 81.40.Vw, 74.62.Fj

\section{Introduction}

In past decades the substantial progress was achieved in preparation of well defined thin films and also in high pressure techniques. The superconductivity of bulk samples under pressure has been already studied in most of elements and many of compounds. However the influence of pressure on superconducting properties of thin films is still not a very well explored field of solid state physics. At ambient pressure $\mathrm{Nb}$ has the highest critical temperature among the elemental superconductors about $9.25 \mathrm{~K}$ [1]. Niobium belongs to $d$-band superconductors with $b c c$ structure. Measurements of superconducting $T_{c}$ of bulk $\mathrm{Nb}$ samples have been already preformed up to $132 \mathrm{GPa}$ using highly sensitive magnetic susceptibility technique with diamond anvil cell [2]. The measured pressure dependence of $T_{c}$ provided evidence for electronic topological transitions in bulk $\mathrm{Nb}$ at $5 \mathrm{GPa}$ and around 60 - $70 \mathrm{GPa}$. Above $70 \mathrm{GPa}$ authors observed steep decrease of $T_{c}$, which was explained by the Fermi level moving to the low density of states region [2]. Thin films of $\mathrm{Nb}$ have been very intensively studied by $\mathrm{S}$. Bose and coworkers in several papers [3-5]. They studied e.g. the influence on superconducting properties of the thickness of thin film, size of grains and other.

\section{Experimental details}

Thin films of $\mathrm{Nb}$ have been prepared by DC magnetron sputtering system (AJA International, USA) in the high vacuum. As a substrate for deposition we used the glass microscope cover slides, which were sonicated in acetone and isopropylalcohol prior to film deposition. We have chosen optimal sputtering conditions to consistently produce films with the highest $T_{c}$ and similar grain size for

*corresponding author; e-mail: gabriel.pristas@saske.sk the $100 \mathrm{~nm}$ film thickness. Typical size of grains was few nms width and $\sim 60 \mathrm{~nm}$ long [6]. Thickness of thin film and size of grains were chosen in order to have properties as similar as possible to bulk sample. Grains with size smaller than $8 \mathrm{~nm}$ are not superconducting due to quantum size effect (QSE) [7]. The residual resistivity ratio (RRR) of the thin film was around 3, which is in good agreement with reports from other research groups [4]. In case of bulk polycrystalline sample the RRR value was about 15 . The electrical contacts on thin film have been realized by four golden wires bonded by ultrasonic contact bonder. Sensitive ac-resistance measurements under hydrostatic pressure up to $30 \mathrm{kbar}$ were carried out in a piston-cylinder pressure cell. As a manometer we used $\mathrm{Pb}$ with $T_{c}=7.19 \mathrm{~K}$. The temperature and magnetic field dependences of resistance at various pressures were performed between 1.6 and $300 \mathrm{~K}$ and in magnetic fields up to $4 \mathrm{~T}$. Magnetic field was oriented perpendicular to thin film surface.

\section{Results and discussion}

Figure 1 shows pressure dependence of superconducting transition for bulk sample of $\mathrm{Nb}$. The width of transition is around $200 \mathrm{mK}$. The $T_{c}$ was estimated as temperature at which the resistance dropped to $50 \%$ of its normal state value (the solid line in Fig. 1). In previous work [8], the measurements of critical temperature $T_{c}$ under pressure were performed using samples with very high RRR (from 500 to 11000) and with very narrow transition (from 3 to $38 \mathrm{mK}$ ). In our measurements the pressure dependence of superconducting transition temperature $T_{c}$ was found to be $\mathrm{d} T_{c} / \mathrm{d} p=-2.5 \mathrm{mK} / \mathrm{kbar}$ for bulk sample, which is in good agreement with result of T.F. Smith [8] $\left(d T_{c} / d p=-2.5 \mathrm{mK} / \mathrm{kbar}\right)$. In case of thin film of $\mathrm{Nb}$ we observed substantially different behavior of $T_{c}$ under pressure. Figure 2 shows pressure dependence of superconducting $T_{c}$ for thin film of $\mathrm{Nb}$. The 


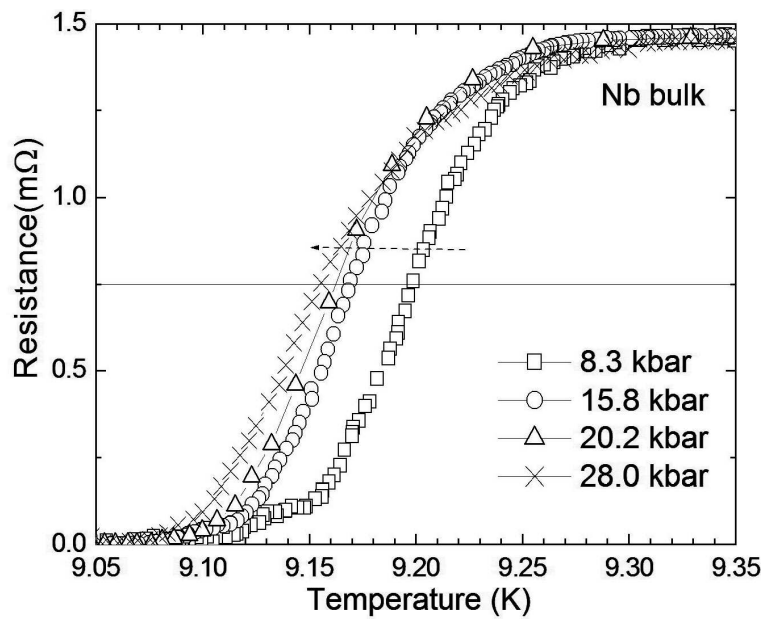

Fig. 1. Temperature dependence of resistance at various pressures for bulk $\mathrm{Nb}$ sample.

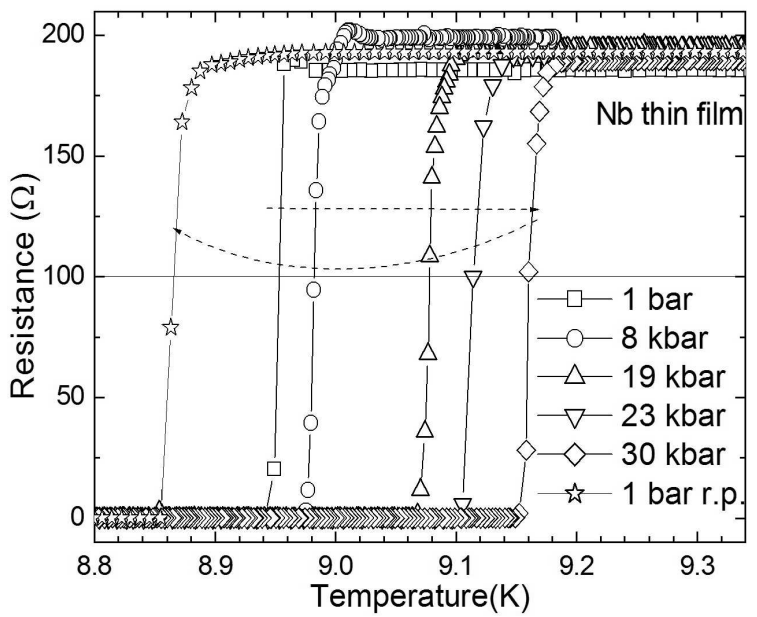

Fig. 2. Temperature dependence of resistance at various pressures for thin film of $\mathrm{Nb}$. Measurement of $R$ vs. $T$ at ambient pressure after releasing of pressure is shown by stars (1 bar r. p.).

criterion for estimation of $T_{c}$ was the same as in previous case. The width of transition is very narrow (about $30 \mathrm{mK}$ ), which points to high purity of sample and also to high homogeneity of the applied pressure. The value of $T_{c}=8.95 \mathrm{~K}$ at ambient pressure is lower than in case of bulk sample $\left(T_{c}=9.25 \mathrm{~K}\right)[1,8]$. With increasing pressure, the superconducting $T_{c}$ increases with the rate of $d T_{c} / d p=7.3 \mathrm{mK} / \mathrm{kbar}$. At maximum pressure of $30 \mathrm{kbar}$ the value of the achieved superconducting transition temperature was $9.15 \mathrm{~K}$, which is close to value of bulk sample at ambient pressure and even higher than for bulk sample at the same pressure.

We suppose, that by application of pressure, the strains induced in thin film during sputtering are released and the properties of thin film get closer to those of bulk sample. After releasing the pressure we measured the superconducting transition again at ambient pressure (see Fig. 2) and surprisingly the $T_{c}$ was lower than for "asprepared" thin film. It will be important to compare microstructure of thin film before and after application of pressure.

Additional measurements under higher pressure are needed to see whether the increase of $T_{c}$ in case of thin film will continue or whether the behavior of $T_{c}$ will follow the behavior of the bulk sample.

\section{Conclusions}

We performed measurements of superconducting transition temperature by electrical resistivity measurements of $\mathrm{Nb}$ thin film as well as the bulk sample under hydrostatic pressure up to $30 \mathrm{kbar}$. We prepared $100 \mathrm{~nm}$ thick niobium thin films, in the high vacuum DC magnetron sputtering system, with $T_{c}=8.95 \mathrm{~K}$ at ambient pressure. We observed an increase of $T_{c}$ with increasing value of pressure $\left(\mathrm{d} T_{c} / \mathrm{d} p=7.3 \mathrm{mK} / \mathrm{kbar}\right)$. On the other side in the case of bulk sample of $\mathrm{Nb}$ we observed a decrease of $T_{c}=9.25 \mathrm{~K}$ value $\left(\mathrm{d} T_{c} / \mathrm{d} p=-2.5 \mathrm{mK} / \mathrm{kbar}\right)$ with the increasing applied pressure. The difference in behavior of thin film and bulk sample comes from the changes of microstructure of the thin film. Measurements under higher pressures are needed.

\section{Acknowledgments}

This work was supported by projects VEGA 2/0135/13, APVV 0036-11, APVV-VVCE 0058, CFNT MVEP project of the Slovak Academy of Sciences, 7th FP EU-Microkelvin and by the EU ERDFITMS26220120005. Liquid nitrogen for the experiments has been sponsored by U.S. Steel Košice, s.r.o.

\section{References}

[1] D.K. Finnemore, T.F. Stromberg, C.A. Swenson, Phys. Rev. 149, 231 (1966).

[2] V.V. Struzhkin, Y.A. Timofeev, R.J. Hemley, H. Mao, Phys. Rev. Lett. 79, 4262 (1997).

[3] S. Bose, P. Raychaudhuri, R. Banerjee, P. Vasa, P. Ayyub, Phys. Rev. Lett. 95, 147003 (2005).

[4] S. Bose, R. Banerjee, A. Genc, P. Raychaudhuri, H.L. Fraser, P. Ayyub, J. Phys.: Condens. Matter 18, 4553 (2006).

[5] S. Bose, P. Raychaudhuri, R. Banerjee, P. Ayyub, Phys. Rev. B 74, 224502 (2006).

[6] G. Pristáš, S. Gabáni, E. Gažo, V. Komanický, M. Orendáč, H. You, Thin Solid Films 556, 470 (2014).

[7] S. Bose, Ch. Galande, S.P. Chockalingam, R. Banerjee, P. Raychaudhuri, P. Ayyub, J. Phys.: Condens. Matter 21, 205702 (2009).

[8] T.F. Smith, Phys. Lett. A 33, 465 (1970). 